\title{
Multiple Magnon Modes and Consequences for the Bose-Einstein Condensed Phase in $\mathrm{BaCuSi}_{2} \mathrm{O}_{6}$
}

\author{
Ch. Rüegg, ${ }^{1}$ D. F. McMorrow, ${ }^{1,2}$ B. Normand, ${ }^{3}$ H. M. Rønnow, ${ }^{4}$ S. E. Sebastian, ${ }^{5}$ I. R. Fisher, ${ }^{5}$ C. D. Batista, ${ }^{6}$ \\ S. N. Gvasaliya, ${ }^{4}$ Ch. Niedermayer, ${ }^{4}$ and J. Stahn ${ }^{4}$ \\ ${ }^{1}$ London Centre for Nanotechnology, Department of Physics and Astronomy, University College London, \\ London WCIE 6BT, United Kingdom \\ ${ }^{2}$ ISIS Facility, Rutherford Appleton Laboratory, Chilton, Didcot OX11 0QX, United Kingdom \\ ${ }^{3}$ Institute of Theoretical Physics, Ecole Polytechnique Fédérale de Lausanne, 1015 Lausanne, Switzerland \\ ${ }^{4}$ Laboratory for Neutron Scattering, ETH Zurich and Paul Scherrer Institute, 5232 Villigen PSI, Switzerland \\ ${ }^{5}$ Geballe Laboratory for Advanced Materials and Department of Applied Physics, Stanford University, \\ Stanford, California 94305, USA \\ ${ }^{6}$ Theoretical Division and MST-NHMFL, Los Alamos National Laboratory, Los Alamos, New Mexico 87545, USA
}

(Received 8 June 2006; published 4 January 2007)

The compound $\mathrm{BaCuSi}_{2} \mathrm{O}_{6}$ is a quantum magnet with antiferromagnetic dimers of $S=1 / 2$ moments on a quasi-2D square lattice. We have investigated its spin dynamics by inelastic neutron scattering experiments on single crystals with an energy resolution considerably higher than in an earlier study. We observe multiple magnon modes, indicating clearly the presence of magnetically inequivalent dimer sites. The more complex spin Hamiltonian revealed in our study leads to a distinct form of magnon BoseEinstein condensate phase with a spatially modulated condensate amplitude.

PACS numbers: 75.10.Jm, 05.30.Jp, 78.70.Nx

The investigation of field-induced quantum phase transitions (QPTs) in magnetic insulators continues to enrich our understanding of the possible quantum ground states of matter [1-5]. Structurally dimerized quantum spin systems play a preeminent role in these studies. One recent example is the phase diagram of the compound $\mathrm{BaCuSi}_{2} \mathrm{O}_{6}$ [6-9], in which antiferromagnetic (AF) dimers formed by the $S=1 / 2$ magnetic moments from pairs of $\mathrm{Cu}^{2+}$ ions are arranged on a quasi-two-dimensional (2D) square lattice. This material is one of the best candidates for the investigation of field-induced Bose-Einstein condensate (BEC) of magnetic quasiparticles, and a 3D-2D dimensional crossover at the QPT has been reported [9].

The ground state of weakly interacting antiferromagnetic dimers is a spin singlet $\left(\left|S, S_{z}\right\rangle=|0,0\rangle\right)$ separated by an energy gap $\Delta$ from excited triplet states $(|1,0\rangle$ and $|1, \pm 1\rangle)$. A QPT occurs at the field $H_{c}=\Delta / g \mu_{B}$, where the $S_{z}=+1$ component condenses. At $H>H_{c}$ the average triplet density becomes finite (magnon BEC) and the ground state changes from a nonmagnetic singlet to an ordered magnetic phase. The triplet quasiparticles may also be considered as hard-core bosons with a kinetic energy and an effective repulsion. Depending on the balance between these terms, a characteristic spatial modulation of the triplet density may arise, whereas in a uniform $\mathrm{BEC}$ this is identical for all sites.

The classes of field-induced QPT known to date in dimer spin systems are summarized in Fig. 1. For magnetic interactions with weak or no frustration, the kinetic energy is dominant and the ordered, or BE-condensed, ground state is uniform at $H>H_{c}$, as in $\mathrm{TlCuCl}_{3}[4,5,10,11]$. In $\mathrm{SrCu}_{2}\left(\mathrm{BO}_{3}\right)_{2}[5,12,13]$ triplet hopping is suppressed by geometrical frustration and the repulsion causes the condensed triplets to form a superlattice with spontaneous breaking of translational symmetry and the appearance of magnetization plateaus [14]. All dimer sites have finite, if weak, triplet density at $H>H_{c}$ [13]. Magnetization plateaus occur also for strong and explicit translational symmetry breaking, which leads to inequivalent dimer sites and multiple magnon modes, as in $\mathrm{NH}_{4} \mathrm{CuCl}_{3}[15,16]$ where the separation of the singlet-triplet gaps exceeds the magnon bandwidths.

Prior to this study the magnetic Hamiltonian used to describe $\mathrm{BaCuSi}_{2} \mathrm{O}_{6}$ was based on an inelastic neutron scattering (INS) investigation with coarse energy resolution [6] and on fits to thermodynamic data [7,8]. Motivated by the exotic low-temperature behavior [9], the lack of high-resolution data and reports of a structural phase transition around $100 \mathrm{~K}[17,18]$, we have investigated the magnetic excitation spectrum by high-resolution INS. We find evidence for explicitly broken translational symmetry in the form of inequivalent dimer sites. Our results therefore establish $\mathrm{BaCuSi}_{2} \mathrm{O}_{6}$ as representative of a fourth category [Fig. 1(IV)] of field-induced QPT system, namely one with a spatially modulated BEC ground state, but without magnetization plateaus, and we propose a closer examination of the magnetic properties around the quantum critical point (QCP).

Five single crystals of $\mathrm{BaCuSi}_{2} \mathrm{O}_{6}$ were coaligned to provide a total mass of $1.25 \mathrm{~g}$. The total mosaic spread of the assembly was excellent and matched the instrumental resolution [Fig. 2(a)]. We note that recently a tetragonal-to-orthorhombic distortion related to a structural modulation with wave vector $\left(\begin{array}{lll}0 & 0.129 & 0\end{array}\right)$ was reported [18]. 

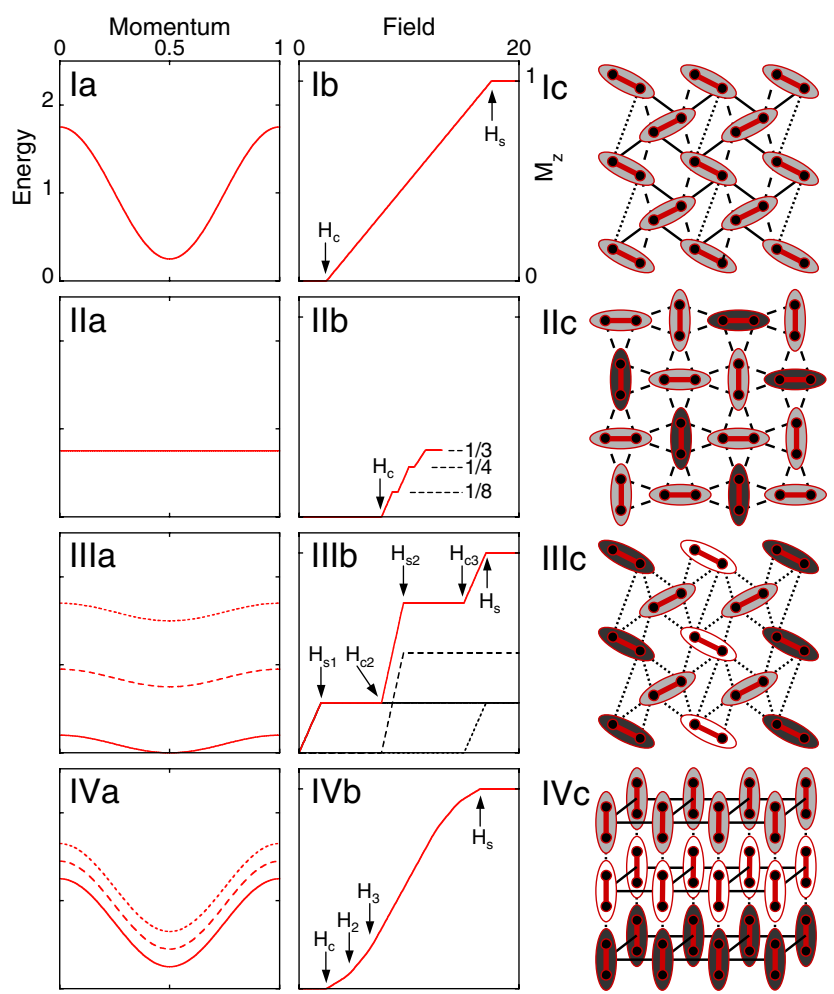

FIG. 1 (color online). Physical properties at a field-induced QPT in dimer-based $S=1 / 2$ spin systems. (a) triplet dispersion at zero field, (b) uniform magnetization $M_{z}$, (c) dimer lattice with triplet condensate amplitude for $H>H_{c}$ represented by increasing gray scale. (I) $3 \mathrm{D}$ systems with uniform magnon BEC, e.g., $\mathrm{TlCuCl}_{3}$. (II) Shastry-Sutherland geometry with magnetization plateaus and strong contrast in condensate amplitude, e.g., $\mathrm{SrCu}_{2}\left(\mathrm{BO}_{3}\right)_{2}$ [field in panel (c) yielding $M_{z}=1 / 3$ ]. (III) Same features for $3 \mathrm{D}$ system with strong translational symmetry-breaking and weak interdimer interactions, e.g., $\mathrm{NH}_{4} \mathrm{CuCl}_{3}$ at $H_{c 2}<H<H_{s 2}$. (IV) Quasi-2D system on square lattice with weakly broken translational symmetry showing no magnetization plateaus, e.g., schematic model for $\mathrm{BaCuSi}_{2} \mathrm{O}_{6}$ at $H_{c}<H<H_{3}$.

INS spectra at $T=1.8 \mathrm{~K}$ and zero magnetic field were collected on the cold-neutron triple-axis spectrometer TASP [19], operated with fixed final momentum $k_{f}=$ $1.3 \AA^{-1}\left(1.5 \AA^{-1}\right)$, a focusing pyrolytic graphite monochromator and analyzer, a cold Be filter, and an open horizontal collimation to gain intensity. The Gaussian energy resolution of $0.14(0.23) \mathrm{meV}$ (FWHM) is considerably improved compared to that in Ref. [6]. Figure 2(b) shows the spectrum at the AF zone center $\boldsymbol{Q}=$ $\left(\begin{array}{lll}q_{h} & q_{k} & q_{l}\end{array}\right)=\left(\begin{array}{lll}0 & 0 & 3\end{array}\right)$ and can be compared directly to Fig. 2(b) of Ref. [6]. Surprisingly, the singlet-triplet gap has a clear multipeak structure over an energy range of $1 \mathrm{meV}$. This splitting cannot be attributed to the multicrystal sample [Fig. 2(a)]. Thus the triplet excitation spectrum of $\mathrm{BaCuSi}_{2} \mathrm{O}_{6}$ displays a much higher degree of intrinsic structure than reported originally.

The INS spectrum in Fig. 2(b) is described by two Gaussian peaks. However, while the excitation at lower energy transfer, denoted as mode I, has an energy width compatible with the instrumental resolution, the peak at higher energies is considerably broader. The evolution of the inelastic signal in the square-lattice plane is presented in Fig. 2(c), and the multipeak structure is observed at every point. At $\left(\begin{array}{lll}0.6 & 0 & 4\end{array}\right)$ the slope of the resolution ellipsoid matches that of the dispersion relation, giving optimal resolution conditions [Fig. 2(d)]: this spectrum reveals that the excitation at higher energy transfer consists of at least two transitions, which are denoted as modes II and III. However, peak II remains broader in energy than expected from the instrumental resolution (cf. mode I), and therefore may consist of more than one mode.

The dispersion of modes I-III is extracted from the data by least-squares fits of the resolved peaks, and is summarized in Figs. 3(a) and 3(b). A cosine dispersion is observed in the square-lattice plane: modes I-III disperse in parallel and are well described by the lowest-order perturbative expression

$$
\begin{aligned}
E_{\alpha}(\boldsymbol{Q})= & J_{\alpha}-J_{\alpha}^{\prime}\left[\cos \left(\pi q_{h}+\pi q_{k}\right)+\cos \left(\pi q_{h}-\pi q_{k}\right)\right] \\
& +J_{\alpha}^{\prime \prime}\left[\cos \left(2 \pi q_{h}\right)+\cos \left(2 \pi q_{k}\right)\right] \\
& +2 J_{\alpha}^{f} \cos \left(\frac{\pi}{2} q_{l}\right)\left|\cos \left(\pi q_{h}\right)-\cos \left(\pi q_{k}\right)\right|
\end{aligned}
$$

where $\alpha=1,2,3$ denotes modes I-III. Parameters $J_{\alpha}$ are intradimer interactions, with $J_{\alpha}^{\prime}$ and $J_{\alpha}^{\prime \prime}$ respectively nearest- and next-nearest-neighbor in-plane interdimer interac-

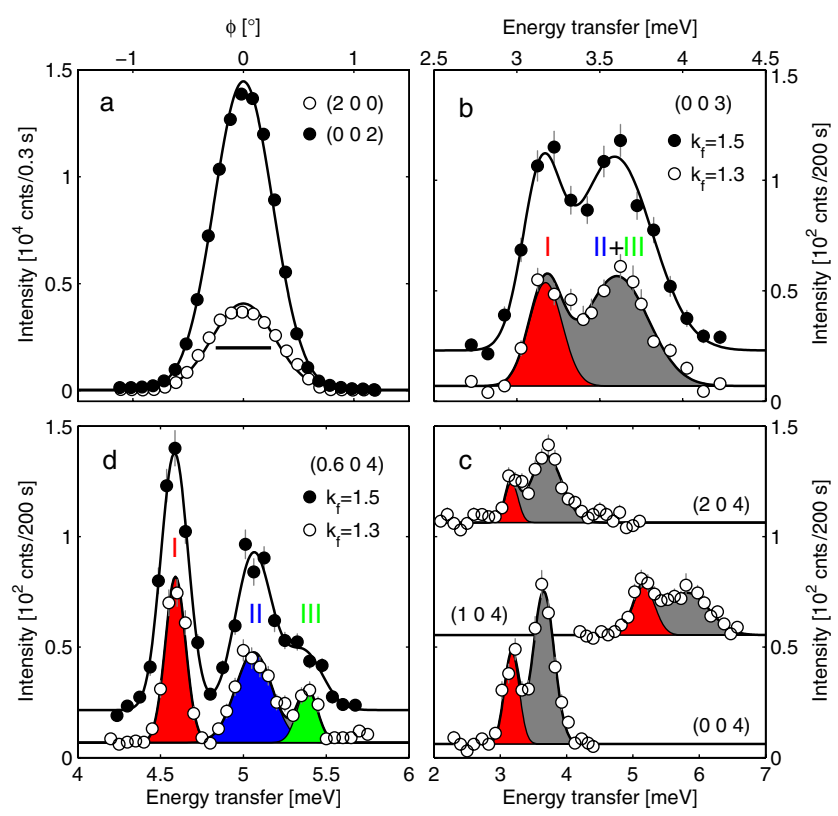

FIG. 2 (color online). (a) Rocking curves across two Bragg peaks of a coaligned $\mathrm{BaCuSi}_{2} \mathrm{O}_{6}$ sample. Horizontal line indicates instrumental resolution (full width) of neutron diffractometer MORPHEUS. (b)-(d) INS spectra of $\mathrm{BaCuSi}_{2} \mathrm{O}_{6}$ at $T=$ $1.8 \mathrm{~K}$ and $H=0 \mathrm{~T}$, experimental conditions as indicated. Momentum vectors in reciprocal lattice units of the tetragonal space group $I 4_{1} / a c d$ with $a=b=10.01 \AA$ and $c=22.47 \AA$ [17], i.e., $\left(\begin{array}{lll}q_{h} & 0 & 4\end{array}\right)$ is the diagonal direction on the square lattice. 
tions and $J_{\alpha}^{f}$ the effective interlayer interactions. The measured energies have no detectable $q_{l}$ dependence (bandwidth below $0.05 \mathrm{meV}$ ), confirming previous claims that $\mathrm{BaCuSi}_{2} \mathrm{O}_{6}$ is quasi-2D. This is a consequence of the frustrated geometry of the interlayer coupling [inset Fig. 3(b)]: the precise determination of $J_{\alpha}^{f}$ is deferred to a future experiment. Here we take $J_{\alpha}^{f}=0$, consistent with the fact that the other parameters in Eq. (1) correspond to decoupled planes. A fit to the complete data set gives dominant intradimer interactions $J_{1}=4.27(1), \quad J_{2}=$ 4.72(1), and $J_{3}=5.04(4)$, with $J_{1}^{\prime}=0.49(1), \quad J_{2}^{\prime}=$ $0.52(1), J_{1}^{\prime \prime}=-0.07(1)$, and $J_{2}^{\prime \prime}=-0.03(1)$, all in $\mathrm{meV}$; we take $J_{3}^{\prime}=\frac{1}{2}\left(J_{1}^{\prime}+J_{2}^{\prime}\right)$ and $J_{3}^{\prime \prime}=\frac{1}{2}\left(J_{1}^{\prime \prime}+J_{2}^{\prime \prime}\right)$ to approximate the dispersion of mode III. Thus we find in contradiction to Ref. [6] that the only significant interdimer interactions are those forming the square lattice, $J_{\alpha}^{\prime} \approx$ $0.1 J_{\alpha}$. The individual gaps at the AF zone center are $\Delta_{1}=$ $3.15(5), \Delta_{2}=3.62(5)$, and $\Delta_{3}=3.94(8) \mathrm{meV}$.

The total INS intensity of modes I-III is modulated along $\left(\begin{array}{lll}0 & 0 & q_{l}\end{array}\right)$ by the dimer structure factor $|f(\boldsymbol{Q})|^{2} \sin \left(d q_{l}\right)^{2}$ [6] [Fig. 3(d)], with $2 d=2.85(6) \AA$ the average projected intradimer separation and $f(\boldsymbol{Q})$ the magnetic form factor of $\mathrm{Cu}^{2+}$. However, fitting intensities I and II + III separately gives $2 d_{\mathrm{I}}=2.52(18) \AA$ and $2 d_{\mathrm{II}+\mathrm{III}}=$ 2.99(10) $\AA$ [Fig. 3(d)], a difference indicative of inequivalent dimer layers. However, a model with uniform layers of dimers oriented parallel to the $c$ axis and modulated only in this direction would predict a weakly decreasing INS in-

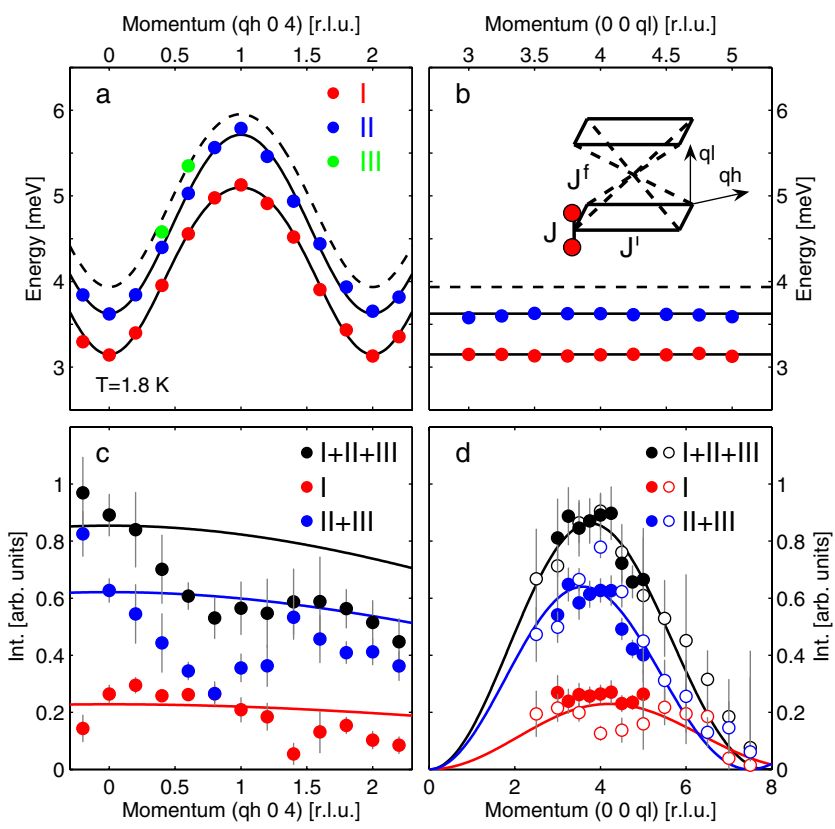

FIG. 3 (color online). (a)-(b) Triplet dispersion in $\mathrm{BaCuSi}_{2} \mathrm{O}_{6}$ at zero field and $T=1.8 \mathrm{~K}$ : (a) in-plane along $\boldsymbol{Q}=\left(\begin{array}{lll}q_{h} & 0 & 4\end{array}\right)$, (b) perpendicular to plane with $\boldsymbol{Q}=\left(\begin{array}{lll}0 & 0 & q_{l}\end{array}\right)$ (see inset). (c)-(d) Momentum-dependence of (scaled) INS intensity, with solid lines as described in text: total intensity modes I-III (black), mode-I (red), and modes II + III (blue) measured with $k_{f}=1.3 \AA^{-1}$ (solid circles) and $1.5 \AA^{-1}$ (open circles). tensity with increasing $\left|\left(\begin{array}{lll}q_{h} & 0 & 4\end{array}\right)\right|$. The $q_{h}$ dependence of the measured intensity [Fig. 3(c)] shows further structure which is clearly inconsistent with equivalent dimer sites in each plane [and thus also with the simple representations in Fig. 1(IVc) and Eq. (1)]. We are driven to conclude that the magnetic interactions are also modulated within the square planes, consistent with what is known of the low-temperature structure [18].

The dependence of the excitation spectrum on magnetic fields up to $H=4 \mathrm{~T}$ is shown in Fig. 4. These data were collected on the spectrometer RITA-II [20] with experimental conditions similar to TASP. The $H=0 \mathrm{~T}$ data in Fig. 4(a) confirm our conclusions concerning the multipeak excitation structure [cf. Fig. 2(d)]. At finite magnetic fields a redistribution of spectral weight is observed. While mode I is clearly split, the transitions at higher energies are barely resolved due to peak overlap [Figs. 4(b) and 4(c)]. One may proceed by fixing the peak widths at the zero-field values and redistributing the spectral weight according to the relative contributions expected for Zeeman-split triplet modes, namely $1 / 4,1 / 2$, and $1 / 4$, respectively, for $S_{z}=+1,0$, and -1 components; the only fitting parameters are the centers of the Gaussian peaks. This approach describes the observed finite-field spectra quite satisfactorily, whereas a model with fieldindependent modes II and III cannot [dashed lines in Figs. 4(b) and 4(c)]. Fits of individual energies, $\tilde{E}_{\alpha}$, and a common $g$ factor are summarized in Fig. 4(d): $\tilde{E}_{1}=$ 4.58(1) $\mathrm{meV}, \tilde{E}_{2}=5.06(1) \mathrm{meV}$, and $\tilde{E}_{3}=5.39(1) \mathrm{meV}$ [cf. Fig. 2(d)], while $g=2.01(4)$, in good agreement with the value reported in Ref. [8]. That each of the modes I-III displays individual Zeeman splitting into components $E_{\alpha}^{ \pm, 0}(H)$ demonstrates again the presence of inequivalent dimer sites. Different candidate mechanisms for a zerofield energy splitting, such as exchange anisotropy or Dzyaloshinskii-Moriya interactions [10,21], are probably present in $\mathrm{BaCuSi}_{2} \mathrm{O}_{6}$ at some small energy scale [22]. However, these would produce only three modes at finite fields and are therefore excluded as the origin of our observations.

The INS results presented here identify $\mathrm{BaCuSi}_{2} \mathrm{O}_{6}$ in a class of low-dimensional quantum magnets which has not yet been considered: despite explicitly broken translational symmetry of the spin Hamiltonian there are no plateaus in the uniform magnetization (Fig. 1) [7]. Both the presence of four dimer layers along the $c$ axis of the large unit cell and the structural distortion at $T \approx 100 \mathrm{~K}$ introduce the potential for different intra- and interdimer interactions. Our results indicate that both interlayer and weak in-plane modulations are present. A minimal magnetic model could be expected to generate at least 4 excitations, consistent with the broad, unresolved appearance of mode II.

The inequivalent dimer sites reflected in the INS spectra result in gaps $\Delta_{\alpha}$ which differ by less than the bandwidths of the individual triplets, in contrast to $\mathrm{NH}_{4} \mathrm{CuCl}_{3}$ [16] [Figs. 1(IIIa,IVa)], removing the possibility of magnetization plateaus [Figs. 1(IIIb,IVb)]. Each of the measured 


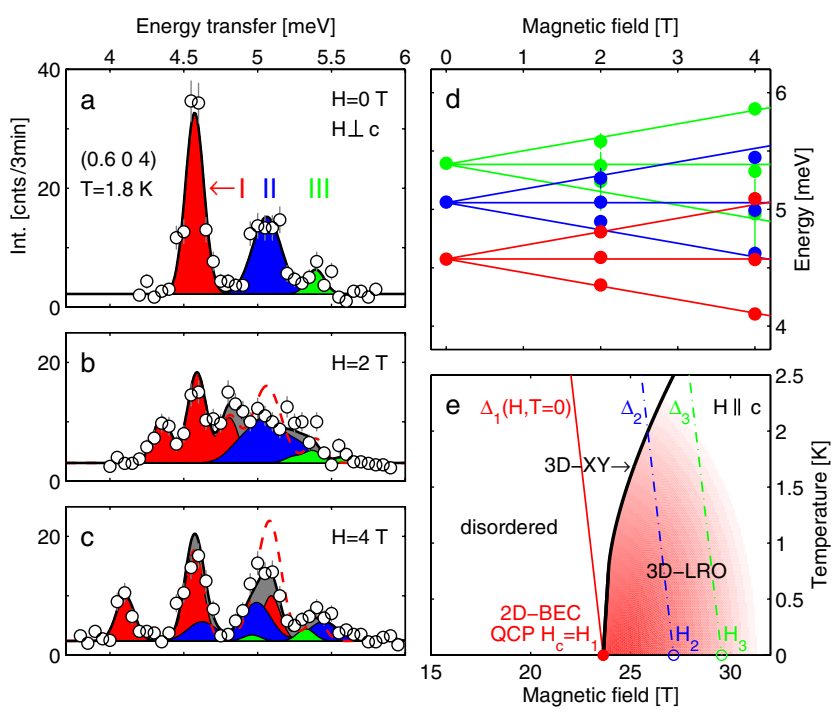

FIG. 4 (color online). (a)-(c) INS spectra at three magneticfield values in $\mathrm{BaCuSi}_{2} \mathrm{O}_{6}$ for $\boldsymbol{Q}=\left(\begin{array}{lll}0.6 & 0 & 4\end{array}\right), T=1.8 \mathrm{~K}$, and $k_{f}=1.3 \AA^{-1}$. (d) Zeeman splitting of triplet modes following procedure described in text. (e) Schematic phase diagram around QPT; intensity of red shading indicates qualitative degree of $c$-axis modulation of BEC order parameter.

modes shows Zeeman splitting in an applied field, which would drive the spin system towards a QCP occurring when the lowest gap $\Delta_{1}$ vanishes at $H_{1}=H_{c}$. Above $H_{c}$, the density of triplet quasiparticles would be largest for sites of type $\alpha=1$, but because of their direct real-space coupling, dimers of types $\alpha=2$ and 3 would also acquire finite, if weak, triplet densities $[13,15]$. The BEC state must therefore be characterized by a spatially modulated amplitude of the order parameter. As the field is increased through the values $H_{\alpha}$ corresponding to the gaps $\Delta_{\alpha}(\alpha=$ 2 and 3), raising the uniform magnetization and the BEC order parameter, the effect of interdimer interactions in a system such as $\mathrm{BaCuSi}_{2} \mathrm{O}_{6}$ is to cause a mixing of the lowlying triplet modes $E_{\alpha}^{+}(H)$ with the ground state. This mixing precludes further phase transitions and causes the magnetization to display a crossover rather than a kink at these fields [Fig. 1(IVb)], in accord with the measurement of Ref. [7]. The spatial modulation of the BEC order parameter may in principle be detected by nuclear magnetic resonance studies of the many inequivalent $\mathrm{Cu}$ sites at $H>H_{c}$ [23].

In $\mathrm{BaCuSi}_{2} \mathrm{O}_{6}$ our results indicate that the dimer modulation occurs predominantly between planes [Figs. 3(c) and $3(\mathrm{~d})$ ], and thus that the indices $\alpha$ correspond to separate bilayers. In this case the triplet density at $H_{c}<H<H_{2}$ is higher on dimer layers $\alpha=1$, and is lower on the intervening bilayers [cf. Fig. 1(IVc)]; indeed the structural modulation must lead in this way to an enhancement of the anisotropic nature of the BEC order parameter, as depicted in Fig. 4(e). The structural modulation also raises the distinct possibility that geometrical frustration of interlayer triplet hopping [Eq. (1)] is less than perfect at low temperatures. However, the thermodynamic measurements of a $2 \mathrm{D} \mathrm{QCP}$ in $\mathrm{BaCuSi}_{2} \mathrm{O}_{6}$ as a consequence of this frustration [9] demonstrate that the energy scale of any unfrustrated hopping component is below $30 \mathrm{mK}$.

In summary, we have investigated the magnetic excitation spectrum of $\mathrm{BaCuSi}_{2} \mathrm{O}_{6}$ in zero field and at $H<H_{c}$ by inelastic neutron scattering on single crystals. The very much higher energy resolution than in earlier studies allows the determination of a spin Hamiltonian whose exchange interactions indicate a complex picture of the ground state and of the field-induced QPT observed in this material. Inequivalent dimer sites give a multimode excitation spectrum, and as a further consequence the BEC phase at $H>H_{c}$ is anticipated to be spatially modulated.

We thank N. Harrison, M. Jaime, and R. Stern for valuable discussions. This work is based on experiments performed at the Swiss spallation neutron source SINQ, Paul Scherrer Institute, Villigen, Switzerland. The project was supported by the Wolfson Foundation, the Swiss National Science Foundation, and the U.S. National Science Foundation, Division of Materials Research, under Grant No. DMR-0134613.

[1] I. Affleck, Phys. Rev. B 43, 3215 (1991).

[2] T. Giamarchi and A. M. Tsvelik, Phys. Rev. B 59, 11398 (1999).

[3] S. Sachdev, Quantum Phase Transitions (Cambridge University Press, Cambridge, England, 1999).

[4] T. Nikuni, M. Oshikawa, A. Oosawa, and H. Tanaka, Phys. Rev. Lett. 84, 5868 (2000).

[5] T. M. Rice, Science 298, 760 (2002).

[6] Y. Sasago, K. Uchinokura, A. Zheludev, and G. Shirane, Phys. Rev. B 55, 8357 (1997).

[7] M. Jaime et al., Phys. Rev. Lett. 93, 087203 (2004).

[8] S. E. Sebastian et al., Phys. Rev. B 72, 100404(R) (2005).

[9] S. E. Sebastian et al., Nature (London) 441, 617 (2006).

[10] Ch. Rüegg et al., Nature (London) 423, 62 (2003).

[11] M. Matsumoto, B. Normand, T. M. Rice, and M. Sigrist, Phys. Rev. Lett. 89, 077203 (2002); Phys. Rev. B 69, 054423 (2004).

[12] H. Kageyama et al., Phys. Rev. Lett. 82, 3168 (1999).

[13] K. Kodama et al., Science 298, 395 (2002).

[14] M. Oshikawa, M. Yamanaka, and I. Affleck, Phys. Rev. Lett. 78, 1984 (1997).

[15] M. Matsumoto, Phys. Rev. B 68, 180403(R) (2003).

[16] Ch. Rüegg et al., Phys. Rev. Lett. 93, 037207 (2004).

[17] K. M. Sparta and G. Roth, Acta Crystallogr. Sect. B 60, 491 (2004).

[18] E. C. Samulon et al., Phys. Rev. B 73, 100407(R) (2006).

[19] F. Semadeni, B. Roessli, and P. Böni, Physica (Amsterdam) 297B, 152 (2001).

[20] K. Lefmann et al., Physica (Amsterdam) 283B, 343 (2000).

[21] V. N. Glazkov, A. I. Smirnov, H. Tanaka, and A. Oosawa, Phys. Rev. B 69, 184410 (2004).

[22] S. E. Sebastian et al., Phys. Rev. B 74, 180401 (2006).

[23] M. Horvatic et al., Prog. Theor. Phys. Suppl. 159, 106 (2005). 